\title{
AVALIAÇÃO DOS RESULTADOS DO TRATAMENTO ARTROSCÓPICO DA EPICONDILITE LATERAL
}

\author{
EVALUATION OF THE RESULTS OF ARTHROSCOPIC \\ TREATMENT OF THE LATERAL EPICONDYLITIS
} Alberto Naoki Miyazaki', Marcelo Fregoneze'2, Pedro Doneux Santos ${ }^{3}$, Luciana Andrade da Silva ${ }^{3}$, Davi Calixto Pires
Jose da Mota Neto

\section{RESUMO}

Objetivo: Avaliar os resultados dos pacientes com epicondilite lateral tratados cirurgicamente pela técnica artroscópica. Métodos: Vinte pacientes foram operados pela técnica artroscópica. A idade variou de 19 a 54 anos (média de 41 anos e oito meses), sendo 12 (60\%) pacientes do sexo feminino e oito (40\%), do masculino. O seguimento mínimo foi de 12 meses e o máximo, de 48 meses, sendo a média de 20 meses. Todos os casos eram refratários ao tratamento conservador (repouso e fisioterapia), com tempo de tratamento clínico prévio variando entre seis e 136 meses. Para a avaliação dos resultados utilizamos os critérios da Associação Médica Americana (AMA), modificados por Bruce. Resultados: Obtivemos 13 (65\%) resultados excelentes e sete (13\%) regulares, com apenas uma complicação (distrofia simpático-reflexa). Este foi o único caso que referiu não estar satisfeito. Conclusão: O tratamento cirúrgico pela técnica artroscópica da epicondilite lateral do cotovelo representa uma boa opção para $65 \%$ dos casos.

Descritores - Artroscopia; Cotovelo de tenista; Avaliação de resultados (Cuidados de Saúde)

\section{ABSTRACT}

Objective: To evaluate the results in patients with lateral epicondylitis surgically treated by the arthroscopy technique. Methods: Twenty patients were submitted to surgery by the arthroscopic technique. Age ranged from 19 to 54 years (average 41 years and eight months). Twelve (60\%) of the patients were female and eight (40\%) male. The minimum follow-up period was 12 months and the maximum period, 48 months, with an average of 20 months. All the cases were refractory to conservative treatment (rest and physiotherapy), with previous clinical treatment times varying ranging from six to 136 months. For the evaluation of the results, we used the criteria of the American Medical Association (AMA), modified by Bruce. Results: We obtained 13 (65\%) excellent results and seven (13\%) regular results, with just one complication (reflex sympathetic dystrophy). This was the only patient who reported dissatisfaction. Conclusion: Surgical treatment by arthroscopy of the lateral epicondylitis of the elbow represents a good option in $65 \%$ of cases.

Keywords - Arthroscopy; Tennis elbow; Outcome assessment (Health Care)

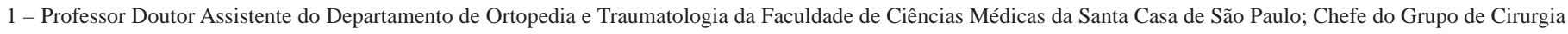
de Ombro e Cotovelo.

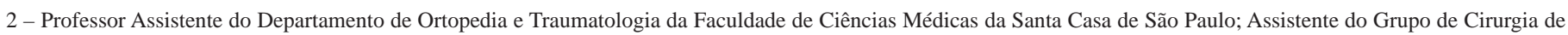
Ombro e Cotovelo.

3 - Assistente do Grupo de Cirurgia de Ombro e Cotovelo.

4 - Médico Estagiário do Grupo de Cirurgia de Ombro e Cotovelo.

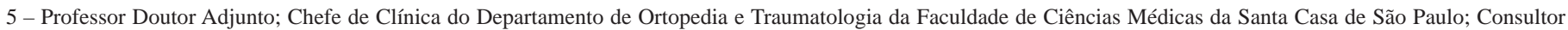
Acadêmico do Grupo de Cirurgia de Ombro e Cotovelo.

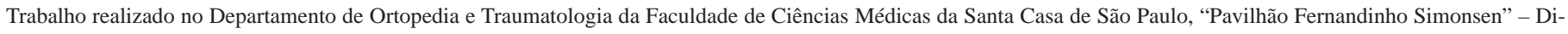
retor: Professor Doutor Osmar Avanzi.

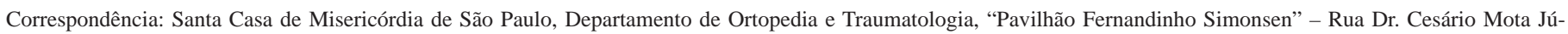
nior, 112 - Vila Buarque - 01220-020 - São Paulo, SP - E-mail: ombro@ombro.med.br - Site: www.ombro.med.br 


\section{INTRODUÇÃO}

A epicondilite lateral (EL) é a afecção mais comum do cotovelo ${ }^{(1)}$, podendo ser desencadeada por trauma ou esforço repetitivo ${ }^{(2)}$. Foi pela primeira vez descrita como doença ocupacional em $1880^{(1,3)}$.

Quanto à etiologia, existem várias teorias para a afecção, incluindo: bursite, sinovite, inflamação ligamentar, periostite e lesão do tendão extensor radial curto do carpo $(\text { ERCC })^{(2)}$.

Em 1979, Nirschl e Pettrone ${ }^{(4)}$ identificaram histologicamente áreas primariamente acometidas pela epicondilite lateral no tendão do ERCC e, em menor grau, na face anteromedial do extensor comum dos dedos (ECD). Essas lesões seriam resultado da aplicação de tração contínua e repetida, levando à microrupturas da origem do ERCC, seguidas de fibrose e formação de tecido de granulação. Macroscopicamente, o tecido apresentava-se com aspecto friável, brilhante e edematoso. Verificaram também que o tendão não estava inflamado, mas degenerado. Por este motivo introduziram o termo hiperplasia angiofibroblástica para descrever o aspecto microscópico da lesão, que é aceito até hoje, assim como sua teoria etiológica ${ }^{(2-4)}$.

A EL acomete mais comumente indivíduos entre 35 e 60 anos, ocorre geralmente no sexo masculino e no membro dominante, sendo mais frequente em brancos $^{(1)}$.

Ao exame físico, o paciente refere dor localizada à palpação na origem dos extensores, muitas vezes determinando com precisão. O ponto máximo de dor pode localizar-se na região anterior e distal ao epicôndilo lateral do úmero ${ }^{(5)}$.

Não é necessária a confirmação diagnóstica por imagem. Se solicitado um exame de ressonância nuclear magnética pode-se observar uma alteração de sinal na origem do tendão ERCC ${ }^{(1)}$. Em 25\% dos pacientes podem aparecer calcificações em tecidos adjacentes ao epicôndilo, principalmente se houver infiltrações prévias de esteroides no local ${ }^{(6)}$.

A maioria dos pacientes responde ao tratamento conservador. Apenas 5 a 10\% evoluem com cronificação dos sintomas ${ }^{(1,7)}$.

Na persistência dos sintomas, pode haver indicação de tratamento cirúrgico. São descritos alguns procedimentos abertos, alguns percutâneos e outros endoscópicos ${ }^{(5,6,8)}$. Baker et $a^{(8)}$ introduziram a liberação artroscópica da origem do tendão ERCC.

O objetivo deste estudo é avaliar o resultado do tratamento cirúrgico artroscópico no alívio da dor causada pela epicondilite lateral, em pacientes refratários ao tratamento não cirúrgico, bem como o retorno a atividades laborativas e esportivas prévias.

\section{CASUÍSTICA E MÉTODOS}

Entre agosto de 1998 e março de 2006, 20 pacientes com epicondilite lateral foram operados pelo Grupo de Ombro e Cotovelo do Departamento de Ortopedia e Traumatologia da Faculdade de Ciências Médicas da Santa Casa de São Paulo, "Pavilhão Fernandinho Simonsen".

Foram incluídos nesta série, retrospectivamente, todos os pacientes com diagnóstico de EL que não melhoraram com o tratamento clínico por, no mínimo, seis meses, e foram submetidos a tratamento cirúrgico artroscópico. Foram considerados critérios de exclusão pacientes que não se enquadraram nos itens descritos acima.

A idade destes pacientes variou de 19 a 54 anos (média de 41 anos e oito meses), sendo 12 (60\%) pacientes do sexo feminino e oito (40\%), do masculino. Todos os pacientes eram destros e o membro dominante foi operado em 13 (65\%) casos. A etiologia da epicondilite lateral foi por esforços repetitivos em 14 (70\%) dos casos, lesão nos esportes em cinco (25\%) e misto, em um (5\%). O seguimento mínimo foi de 12 meses e o máximo, de 48 meses, sendo a média de 20 (Tabela 1).

Os pacientes foram tratados primariamente com repouso e fisioterapia. Treze (65\%) pacientes foram submetidos à infiltração de corticoide, sendo no mínimo uma infiltração e no máximo três, com a média de duas. Um paciente foi submetido a tratamento cirúrgico prévio por via aberta realizado em outro serviço havia 11 anos, com melhora completa dos sintomas, porém, apresentou recidiva do quadro após nove anos. O tempo de tratamento clínico variou de seis meses a 136 meses, com média de 28,5 meses (Tabela 1).

Os pacientes foram operados em posição de decúbito ventral, sendo realizado primeiramente o portal posterolateral entre o olecrano e o epicôndilo lateral para posicionamento do artroscópico. Em seguida, realizou-se o inventário do compartimento posterior e tratou-se qualquer lesão que estivesse presente ali (casos 6, 7, 14, 17 e 20). Por um portal anteromedial proximal explorou-se o compartimento anterior e localizou-se, sob visão direta, o melhor posicionamento do portal anterolateral para introdução da lâmina artroscópica para partes moles (Figura 1). Iniciou-se, por este portal, a ressecção parcial da cápsula anterolateral, a identificação e ressecção do tecido angiofibroblástico, além da desinserção parcial da musculatura extensora. Em todos ao casos, foi realizada uma descorticação da região anterior do epicôndilo lateral com a broca artroscópica (Figura 2). 
Tabela 1 - Dados clínicos dos pacientes

\begin{tabular}{|c|c|c|c|c|c|c|c|c|c|c|c|c|c|c|c|c|}
\hline & \multirow{2}{*}{ Idade } & \multirow{2}{*}{ Sexo } & \multirow{2}{*}{ Dominância } & \multirow{2}{*}{ Profissão } & \multirow{2}{*}{ Esporte } & \multirow{2}{*}{$\begin{array}{c}\text { Tto } \\
\text { clínico }\end{array}$} & \multirow{2}{*}{ Infiltração } & \multirow{2}{*}{$\begin{array}{c}\text { Cirurgia } \\
\text { prévia }\end{array}$} & \multirow{2}{*}{$\begin{array}{c}\text { Tempo de } \\
\text { seguimento }\end{array}$} & \multirow{2}{*}{$\begin{array}{c}\text { Lesões } \\
\text { intra- } \\
\text { articulares }\end{array}$} & \multicolumn{5}{|c|}{ BRUCE } & \multirow{2}{*}{ Complicações } \\
\hline & & & & & & & & & & & ADM & AVD & Dor & Anatomia & Total & \\
\hline 1 & 50 & M & + & Almoxarife & & $17 \mathrm{~m}$ & & & $12 \mathrm{~m}$ & & 60 & 20 & 15 & 5 & 100 & \\
\hline 2 & 44 & $\mathrm{~F}$ & & Operadora & & $16 \mathrm{~m}$ & 1 & & $12 \mathrm{~m}$ & & 60 & 20 & 15 & 5 & 100 & \\
\hline 3 & 47 & $\mathrm{~F}$ & + & Analista contábil & & $41 \mathrm{~m}$ & 2 & & $13 \mathrm{~m}$ & & 60 & 20 & 13 & 5 & 98 & \\
\hline 4 & 31 & M & + & Dentista & Tênis & $7 \mathrm{~m}$ & 1 & & $18 \mathrm{~m}$ & & 60 & 20 & 15 & 5 & 100 & \\
\hline 5 & 46 & M & + & Caldereiro & & $136 \mathrm{~m}$ & & & $18 \mathrm{~m}$ & & 60 & 20 & 15 & 5 & 100 & \\
\hline 6 & 41 & $\mathrm{~F}$ & + & Do lar & & $17 \mathrm{~m}$ & & & $26 \mathrm{~m}$ & HPS & 60 & 20 & 15 & 5 & 100 & \\
\hline 7 & 26 & M & & Analista financeiro & Vôlei & $13 \mathrm{~m}$ & 2 & & $18 \mathrm{~m}$ & HPS & 60 & 20 & 15 & 5 & 100 & \\
\hline 8 & 30 & M & + & Engenheiro & Tênis & $11 \mathrm{~m}$ & & & $18 \mathrm{~m}$ & & 60 & 20 & 15 & 5 & 100 & \\
\hline 9 & 53 & M & + & Engenheiro & Tênis & $36 \mathrm{~m}$ & & & $30 \mathrm{~m}$ & & 60 & 20 & 15 & 5 & 100 & \\
\hline 10 & 54 & $\mathrm{~F}$ & + & Aux. de limpeza & & $42 \mathrm{~m}$ & 2 & & $13 \mathrm{~m}$ & & 60 & 20 & 13 & 5 & 98 & \\
\hline 11 & 52 & M & + & Advogado & Tênis & $14 \mathrm{~m}$ & 2 & & $24 m$ & & 60 & 20 & 15 & 5 & 100 & \\
\hline 12 & 43 & $\mathrm{~F}$ & & Do lar & & $22 \mathrm{~m}$ & 1 & & $38 \mathrm{~m}$ & & 60 & 20 & 15 & 5 & 100 & \\
\hline 13 & 45 & $\mathrm{~F}$ & + & Analista de sistemas & & $72 \mathrm{~m}$ & 2 & & $13 m$ & & 60 & 20 & 15 & 5 & 100 & \\
\hline 14 & 37 & $\mathrm{~F}$ & & Aux. enfermagem & & $25 \mathrm{~m}$ & & & $18 \mathrm{~m}$ & HPS & 54 & 15 & 10 & 5 & 84 & \\
\hline 15 & 44 & M & & Motorista & Pesca & $46 m$ & & & $12 m$ & & 60 & 15 & 5 & 5 & 85 & \\
\hline 16 & 45 & $\mathrm{~F}$ & & Aux. de limpeza & & $7 \mathrm{~m}$ & 3 & & $26 \mathrm{~m}$ & & 60 & 10 & 10 & 5 & 85 & \\
\hline 17 & 46 & $\mathrm{~F}$ & + & Aux. enfermagem & & $24 \mathrm{~m}$ & 3 & sim & $48 \mathrm{~m}$ & HPS & 60 & 15 & 5 & 5 & 85 & \\
\hline 18 & 38 & $\mathrm{~F}$ & + & Cozinheira & & $10 \mathrm{~m}$ & 2 & & $13 \mathrm{~m}$ & & 60 & 10 & 5 & 5 & 80 & DSR \\
\hline 19 & 44 & $\mathrm{~F}$ & & Aux. de limpeza & & $6 \mathrm{~m}$ & 3 & & $18 \mathrm{~m}$ & & 60 & 15 & 5 & 5 & 85 & \\
\hline 20 & 19 & $\mathrm{~F}$ & + & Estudante & & $12 \mathrm{~m}$ & 2 & & $12 \mathrm{~m}$ & HPS & 60 & 15 & 5 & 5 & 85 & \\
\hline
\end{tabular}

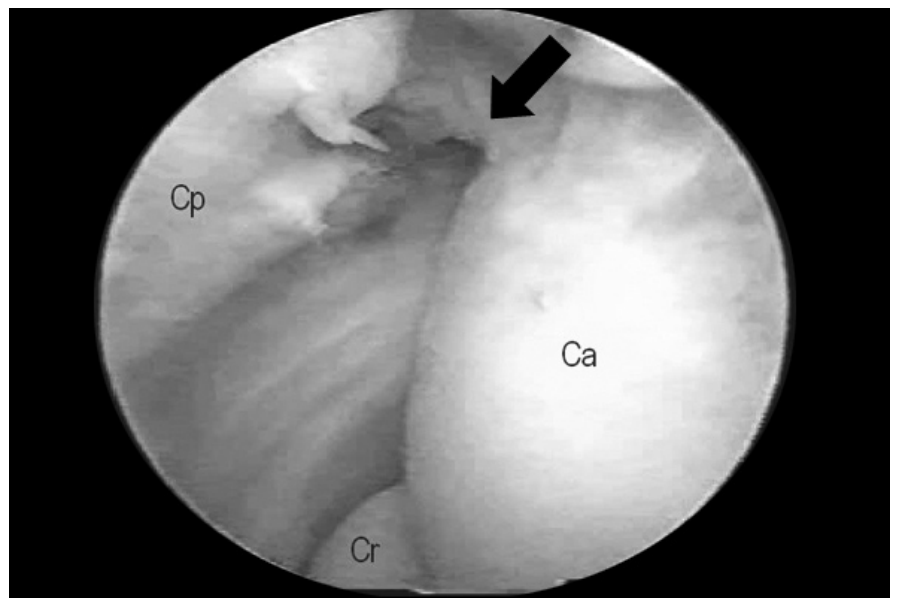

Figura 1 - Imagem de artroscopia do cotovelo direito mostrando: $\mathrm{Cp}=$ cápsula articular anterolateral, $\mathrm{Ca}=$ capítulo, $\mathrm{Cr}=$ cabeça do rádio e tecido angiofibroblástico (seta)

A avaliação pós-operatória foi realizada com duas, quatro e seis semanas e três, seis e 12 meses. Nas duas primeiras semanas, foram prescritos analgésicos e os pacientes foram mantidos com o membro superior operado na tipoia. A movimentação ativa pós-operatória foi incentivada. Não foi indicada fisioterapia específica.

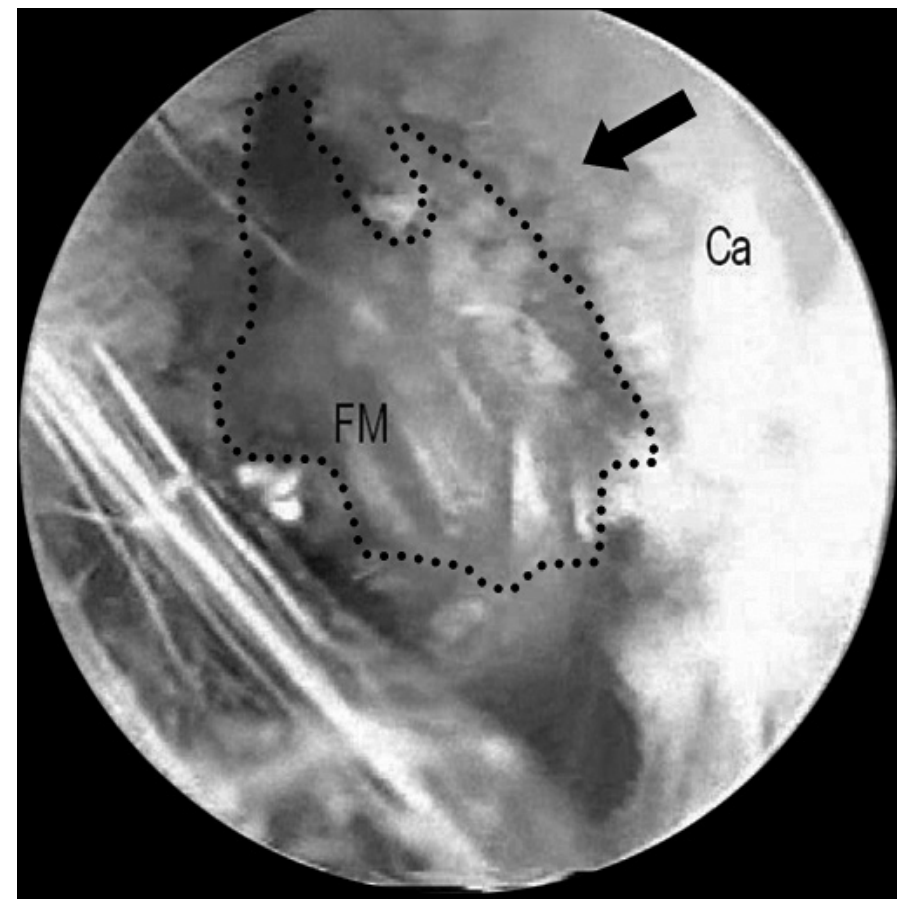

Figura 2 - Imagem de artroscopia do cotovelo direito mostrando: $\mathrm{FM}$ = fáscia muscular, $\mathrm{Ca}=$ capítulo, tecido angiofibroblástico (seta) e área pontilhada = área de tecido angiofibroblástico desbridado 
Para avaliação dos resultados usamos os critérios da Associação Médica Americana (AMA), modificados por Bruce et $a l^{(9)}$ (Quadro 1).

\section{Quadro 1 - Critérios da AMA modificados por Bruce et al(8)}

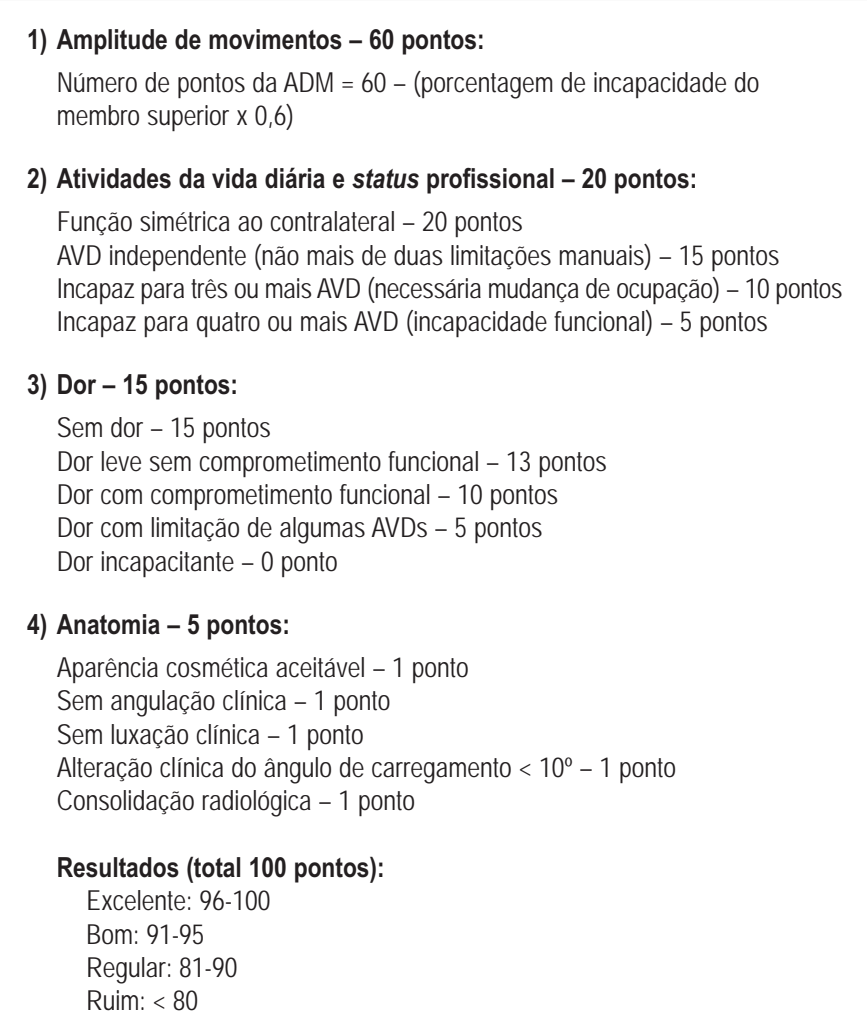

Legenda: AMA: Associação Médica Americana, ADM: amplitude de movimento, AVD: atividades da vida diária

Os resultados foram comparados estatisticamente com as variáveis: sexo, profissão e prática esportiva. Para tanto usamos o programa SPSS (Statistical Package for Social Sciences), em sua versão 13.0, para a obtenção dos resultados e aplicação do Teste Exato de Fisher, com o intuito de verificarmos o grau de associação entre as variáveis de interesse. Adotamos o nível de significância de 5\% (0,05), para a validação dos resultados.

\section{RESULTADOS}

Dos 20 pacientes operados pela técnica artroscópica encontramos 13 (65\%) resultados excelentes e sete (35\%) regulares. Apenas um paciente perdeu pontos no critério amplitude de movimento (Tabela 1). Um paciente apresentou, como complicação, distrofia simpático-reflexa (DSR) (caso 18). Ao serem questionados sobre a satisfação com os resultados da cirurgia, apenas o paciente que evoluiu com DSR relatou não estar satisfeito.
Apenas um paciente (caso número 17, Tabela 1) foi submetido a cirurgia prévia por via aberta em outro serviço. Evoluiu bem por nove anos, quando voltou a apresentar sintomas. Apesar do tratamento clínico intermitente por dois anos, não houve melhora dos sintomas, tendo sido submetido, então a tratamento artroscópico, evoluindo com resultado regular.

O resultado estatístico do estudo de associação entre as variáveis 'sexo/resultado', 'profissão/resultado' e 'prática esportiva/resultado' mostrou os respectivos valores: $\mathrm{p}=0,158, \mathrm{p}=0,158$ e $\mathrm{p}=0,354$, sendo, portanto, não significantes estatisticamente.

\section{DISCUSSÃO}

As controvérsias sobre o tratamento cirúrgico da EL permanecem até os dias de hoje. A técnica artroscópica possibilita a excisão da origem dos tendões extensores envolvidos na doença, e a visualização e tratamento de lesões intra-articulares associadas, podendo promover um retorno precoce às atividades ${ }^{(6,10)}$.

Bons resultados têm sido relatados com técnicas abertas tradicionais, mas elas não localizam lesões intraarticulares associadas, que estão presentes em $11 \%$ a $69 \%$ dos $\operatorname{casos}^{(4,6,10)}$.

Em nossa série encontramos lesões intra-articulares em $25 \%$ dos pacientes, sendo compatível com a literatura, e pudemos tratá-las de imediato, durante o mesmo procedimento.

Obtivemos 65\% de ótimos resultados, compatível com alguns trabalhos da literatura em que foram encontrados $62 \%$ de pacientes relativamente livres de dor e, $10 \%$ que, apesar de satisfeitos, mantinham dor em algumas atividades da vida diária ${ }^{(6)}$. Cohen e Romeo ${ }^{(1)}$ constataram presença de dor de intensidade moderada a grave em $24 \%$ dos pacientes após um ano de cirurgia aberta e/ou artroscópica. Com dois anos, 33\% apresentaram resultados regulares e ruins ${ }^{(1)}$. Em cirurgia aberta, Verhaar et al $^{(11)}$ também apresentaram resultados semelhantes com $69 \%$ de pacientes satisfeitos sem dor ou com dor leve às atividades, assim também Nirschil et $a l^{(4)}$ obtiveram $97,7 \%$ de relatos de melhora da dor, apesar de somente $85,2 \%$ dos pacientes terem conseguido retomar suas atividades completamente.

Os 13 (65\%) pacientes classificados como resultado excelente, estavam satisfeitos com o tratamento. Dos sete (35\%) que evoluíram com resultados regulares, seis (30\%) também estavam satisfeitos com sua evolução, mas não conseguiram retomar, completamente, às suas atividades. 
Tivemos um paciente (5\%) que evoluiu com distrofia simpático-reflexa do membro operado, considerada uma complicação, e ele não está satisfeito com o resultado (Tabela 1).

$\mathrm{Na}$ literatura encontramos estudos que demonstram até $100 \%$ de ótimos resultados com a técnica artroscópica, o que não ocorreu $\operatorname{conosco}^{(6,7)}$.

Levando-se em consideração a dor como critério de avaliação, observamos que nossos resultados não são semelhantes aos encontrados na literatura. A ausência de uma escala de avaliação específica do resultado do tratamento da epicondilite ${ }^{(7)}$ e a diferença entre os critérios de avaliação utilizados em vários estudos podem justificar este resultado. Podemos entender que a qualificação regular em seis (30\%) de nossos casos seria explicada devido ao critério por nós adotado, que é mais rigoroso quando avalia as atividades da vida diária (Tabela 1). O paciente número 17 , que havia sido submetido a cirurgia prévia havia 11 anos, permaneceu assintomático por nove anos antes de manifestar novamente o quadro, porém, não acreditamos que tal fato tenha influenciado na recidiva, nem no resultado do tratamento atual.

Os pacientes que apresentam lesão que possibilite o afastamento do trabalho, segundo a literatura, tendem a ter pior evolução nos critérios: atividades da vida diária, trabalho e/ou esporte quando comparados com esportistas $^{(4,6,11)}$. Em nosso estudo, no grupo de sete pacientes classificados como regulares, seis pacientes estavam afastados de suas funções recebendo auxílio doença. Mesmo não sendo estatisticamente significante, observamos melhores resultados com relação ao retorno às atividades laborais e/ou esportivas, nos pacientes cuja etiologia da epicondilite estava associada à prática esportiva ${ }^{(7,10,12)}$.

Concordamos com Morrey ${ }^{(13)}$ e acreditamos que a persistência da dor pode ter ocorrido por uma escolha errada do paciente a ser operado (causas relacionadas ao trabalho), um erro diagnóstico inicial, ou mesmo porque as alterações provocadas pela doença não foram totalmente corrigidas durante a cirurgia. Os casos que não obtiveram um bom resultado precisam ser reavaliados.

Excluindo os pacientes que não obtiveram ganhos secundários com a doença podemos notar que o tratamento artroscópico da EL ofereceu uma série de vantagens: pôde-se avaliar doenças intra-articulares e tratá-las; fezse o desbridamento do tendão do ERCC sem dividir as fibras da aponeurose do ECD; o período de reabilitação foi curto; e, ainda, houve a possibilidade de adicionar um procedimento aberto, caso tivesse havido necessidade, o que não foi observado. Isso foi exatamente o que observou Cohen e Romeo ${ }^{(1)}$ e Baker et al ${ }^{(6)}$ em seus respectivos trabalhos.

As desvantagens relatadas do método endoscópico são o risco de lesão neurovascular no momento em que se faz os portais $^{(14)}$, e a lesão ligamentar posterolateral do cotovelo. Este tipo de lesão pode ser uma das causas de falha do tratamento cirúrgico da doença ${ }^{(10,13,14)}$. Estas complicações são raras e não ocorreram no nosso estudo.

\section{CONCLUSÃO}

O tratamento cirúrgico pela técnica artroscópica da epicondilite lateral do cotovelo representa uma boa opção, sendo satisfatória em $65 \%$ dos casos.

\section{REFERÊNCIAS}

1. Cohen MS, Romeo AA. Lateral epicondylitis: open and arthroscopic treatment. J Hand Surg Am. 2001;3(1):172-6.

2. Owens BD, Murphy KP, Kuklo TR. Arthroscopic release for lateral epicondylitis. Arthroscopy. 2001;17(6):582-7.

3. Nirschl RP. Lateral tennis elbow. Tech Shoulder Elbow Surg. 2000; 1(3):192-200.

4. Nirschl RP, Pettrone FA. Tennis elbow: the surgical treatment of lateral epicondylitis. J Bone Joint Surg Am. 1979;61(6):832-9.

5. Lech O, Piluski PCF, Severo AL. Epicondilite lateral do cotovelo. Rev Bras Ortop. 2003;38(8):421-35.

6. Baker CL Jr, Murphy KP, Gottlob CA, Curd DT. Arthroscopic classification and treatment of lateral epicondylitis: two-year clinical results. J Shoulder Elbow Surg. 2000;9(6):475-82.

7. Zoppi Filho A, Vieira LAG, Ferreira Neto AA, Benegas E. Tratamento artroscópico da epicondilite lateral do cotovelo. Rev Bras Ortop. 2004;39(3):93-101.

8. Baker CL Jr, Shalvoy RM. The prone position for elbow arthroscopy. Clin Sports Med. 1991;10(3):623-8.

9. Bruce HE, Harvey JP, Wilson JC Jr. Monteggia fractures. J Bone Joint Surg Am. 1974;56(8):1563-76.

10. Smith AM, Castle JA, Ruch DS. Arthroscopic resection of the common extensor origin: anatomic considerations. J Shoulder Elbow Surg. 2003;12(4):375-9.

11. Verhaar J, Walenkamp G, Kester A, van Mamerem H, van der Linden T. Lateral extensor release for tennis elbow: a prospective long-term follow-up study. J Bone Joint Surg Am. 1993;75(7):1034-43.

12. Coonrad RW, Hooper WR. Tennis elbow: its course, natural history, conservative and surgical management. J Bone Joint Surg Am. 1973;55(6):1177-82.

13. Morrey BF. Reoperation for failed surgical treatment of refractory lateral epicondylitis. J Shoulder Elbow Surg. 1992;1(1):147-55.

14. Kalainov DM, Cohen MS. Posterolateral rotatory instability of the elbow in association with lateral epicondylitis. A report of three cases. J Bone Joint Surg Am. 2005;87(5):1120-5. 